\title{
COMPARATIVE ACTIVITY OF DORIPENEM, IMIPENEM AND MEROPENEM AGAINST GRAM NEGATIVE PATHOGENS: A PRELIMINARY STUDY
}

\author{
Vipin Sam Alexander', Aroma Oberoi², Atul Kumar ${ }^{3}$ \\ ${ }^{1}$ Assistant Professor, Department of Microbiology, Christian Medical College, Ludhiana. \\ 2Professor and Head, Department of Microbiology, Christian Medical College, Ludhiana. \\ ${ }^{3}$ Post Graduate Resident, Department of Microbiology, Christian Medical College, Ludhiana.
}

\section{ABSTRACT}

\section{BACKGROUND AND OBJECTIVE}

Doripenem is a new parenteral carbapenems, which has beta-lactamase stability and is not inactivated by renal dehydropeptidases. Doripenem has a spectrum of activity similar to imipenem and ertapenem against Gram-positive cocci and similar to meropenem against Gram-negative pathogens. In this study, we summarize the activity of doripenem against Gram negative bacilli in comparison with other carbapenems (Imipenem, meropenem) and select group of antimicrobial drugs by disk diffusion.

\section{SETTINGS AND DESIGN}

A retrospective study was conducted over a period of 3 months (December 2013 to February 2014) in the Department of Microbiology of a tertiary care hospital in Northern India.

\section{METHODS AND MATERIAL}

Gram negative bacillary isolates were subjected to antimicrobial susceptibility with the following antibiotics: imipenem, meropenem, doripenem, ceftazidime, ceftriaxone, amikacin, ciprofloxacin, piperacillin/tazobactam, and trimethoprimsulphamethoxazole by employing the Kirby-Bauer disk diffusion method. The results were interpreted as per CLSI guidelines.

\section{RESULTS}

A total of 498 isolates obtained from urine, skin and soft tissue specimens and lower respiratory specimens were included in the study. The most frequent Gram-negative bacilli isolated were E. coli (31.5\%), Acinetobacter spp. (20.1\%), Klebsiella spp. (19.5\%), P. aeruginosa (16.7\%), Enterobacter spp. (8.2\%), Proteus spp. (3\%) and Citrobacter spp. (1\%). The isolates showed highest rates of susceptibility to meropenem (65.5\%) followed by imipenem (63.7\%), doripenem (55.8\%), amikacin (53.4\%), piperacillin/tazobactam (48.7\%), trimethoprim-sulphamethoxazole (38.3\%), ceftazidime (26.9\%), ceftriaxone (23.9\%) and ciprofloxacin $(25.3 \%)$.

\section{CONCLUSIONS}

In this study, the activity of doripenem was found to be lower than meropenem and imipenem against all the isolates tested. Further detailed evaluation of doripenem is required with in-vitro MIC studies and their correlation with clinical outcomes.

\section{KEYWORDS}

Doripenem, Carbapenemases, Gram Negative Bacilli.

HOW TO CITE THIS ARTICLE: Alexander VS, Oberoi A, Kumar A. Comparative activity of doripenem, imipenem and meropenem against gram negative pathogens: a preliminary study. J. Evolution Med. Dent. Sci. 2016;5(44):2758-2762,

DOI: $10.14260 /$ jemds/2016/644

\section{INTRODUCTION}

Carbapenems are a class of antimicrobials that are structurally related to penicillin. They continue to be one of the most active classes of antibiotics against many resistant pathogens. However, resistance to carbapenems is steadily increasing over the last decade according to various reports.(1) Doripenem (Formerly S-4661) is a new parenteral carbapenems, which has beta-lactamase stability and is not inactivated by renal dehydropeptidases.

Financial or Other, Competing Interest: None.

Submission 28-03-2016, Peer Review 11-05-2016,

Acceptance 17-05-2016, Published 02-06-2016.

Corresponding Author:

Dr. Aroma Oberoi,

Professor and Head,

Department of Microbiology,

Christian Medical College,

Ludhiana, Punjab.

E-mail: draromaoberoi@yahoo.com

DOI: $10.14260 /$ jemds/2016/644
Doripenem binds to and inactivates Penicillin Binding Proteins (PBP) like other carbapenems, thus inhibiting bacterial cell wall synthesis and causing cell death. Doripenem binds to PBP2 and PBP3 in P. aeruginosa, PBP2 in Escherichia coli. PBP1, PBP2 and PBP4 of Staphylococcus aureus.(2) Doripenem has a spectrum of activity similar to imipenem and ertapenem against Gram-positive cocci, and similar to meropenem against Gram-negative pathogens. $(3,4)$ Doripenem was recently approved by the Food and Drug Administration to treat complicated intra-abdominal infections and complicated urinary tract infections including pyelonephritis caused by susceptible bacteria. In this study, we summarize the activity of doripenem in comparison with other carbapenems (Imipenem, meropenem) and select group of antimicrobial drugs by disk diffusion.

\section{MATERIALS AND METHODS}

The study was conducted in the Microbiology Department of a tertiary care teaching hospital in North India. A retrospective 
analysis of 498 gram negative bacterial isolates was conducted over a period of three months from December 2013 to February 2014. Consecutive, non-duplicate isolates from patients visiting the outpatient department as well as patients admitted to surgical, medical and ICU wards were included in the study. The isolates were predominantly obtained from patients with documented respiratory tract, skin and soft tissue and urinary tract infections. Blood agar, chocolate agar and MacConkey agar plates were used as the primary plating media. Samples were processed and isolates were identified by standard microbiological techniques. ${ }^{(5)}$

\section{Antimicrobial Susceptibility Testing}

Antimicrobial susceptibility testing was performed by the Kirby-Bauer disk diffusion method on Mueller-Hinton agar. The isolates were tested with the following antibiotics (HiMedia Laboratories, Mumbai, India): Ceftazidime (30 mcg), Amikacin (30 mcg), Ciprofloxacin (5 $\mathrm{mcg})$, Piperacillin/Tazobactam (100/10 mcg), Imipenem (10 mcg), Doripenem (10 mcg), Meropenem (10 $\mathrm{mcg})$ and Trimethoprim-sulphamethoxazole $(1.25 / 23.75 \mu \mathrm{g})$. The antimicrobial susceptibility pattern was interpreted as per the Clinical and Laboratory Standards Institute (CLSI) guidelines. (6) E. coli ATCC 25922 and Pseudomonas aeruginosa ATCC 27853 were used for the quality control.

\section{RESULTS}

A total of 498 isolates were included as part of the study. These isolates were obtained from the following samples: urine (35.1\%), soft tissue specimens (28.1\%), lower respiratory specimens (29.8\%) and other samples (7\%) like cerebrospinal fluid, ascitic fluid, central line tip, external prostatic secretions. The most frequent Gram-negative bacilli collected were E. coli (31.5\%), Acinetobacter spp. (20.1\%), Klebsiella spp. (19.5\%); P. aeruginosa (16.7\%); Enterobacter spp. (8.2\%); Proteus spp. (3\%) and Citrobacter spp. (1\%).

Table 1 shows the susceptibility rates of doripenem and comparator agents against all the Gram negative bacterial isolates. The isolates showed highest rates of susceptibility to carbapenems with $65.5 \%$ of the isolates being susceptible to meropenem followed by imipenem (63.7\%) and doripenem $(55.8 \%)$. Among the other antimicrobials tested, $48.7 \%$ of the isolates were susceptible to piperacillin/tazobactam. The isolates showed lowest rates of susceptibility to ceftriaxone (23.9\%) and ciprofloxacin (25.3\%).

The species wise in vitro activity of doripenem in comparison with comparator agents is shown in Table 2. The activity of doripenem against E. coli was comparable to imipenem and meropenem. Susceptibility rates to amikacin $(82.8 \%)$ and piperacillin/tazobactam (75\%) were also found to be high, while the activities of cephalosporins, ciprofloxacin and trimethoprim-sulphamethoxazole were found to be variable. The susceptibility rates of Klebsiella spp. to doripenem was $40.2 \%$, when compared to meropenem (64.9\%) and imipenem (59.8\%). Trimethoprimsulphamethoxazole was the most active antimicrobial agent against Klebsiella spp. with susceptibility rates of $93.8 \%$.
Against Enterobacter spp., the potency of doripenem was comparable with imipenem with $75.6 \%$ of the isolates being susceptible, while meropenem exhibited lower activity (68.3\%).

Among P. aeruginosa isolates, $53 \%$ were sensitive to doripenem, $51.8 \%$ to imipenem and $50.6 \%$ to meropenem. Amikacin was the most active drug against P. aeruginosa with $59 \%$ of the isolates being sensitive. Resistance to carbapenems was most pronounced amongst Acinetobacter spp. with only $14.1 \%$ of the isolates being susceptible to doripenem, $33 \%$ to imipenem and $31 \%$ to meropenem. Table 3 shows the antimicrobial susceptibility rates of doripenem and other antimicrobial agents against Gram negative bacilli isolated from Intensive Care Units (ICU).

The activities of doripenem and other antimicrobials against cephalosporin-susceptible and resistant Gram negative bacilli are presented in the Figure. Among E. coli isolates, doripenem had susceptibility rates of $100 \%$ and $76.2 \%$ against ceftazidime-susceptible and non-susceptible isolates respectively, compared to meropenem $(100 \%$ and $70.5 \%$, respectively) and imipenem $(100 \%$ and $79.1 \%$ respectively). In Klebsiella spp., doripenem had susceptibility rates of $100 \%$ and $29.6 \%$ against ceftazidime-susceptible and non-susceptible isolates respectively, compared to meropenem (87.5\% and $61.7 \%$ respectively) and imipenem (100\% and $53.1 \%$ respectively).

In Enterobacter spp., doripenem had susceptibility rates of $100 \%$ and $65.5 \%$ against ceftazidime-susceptible and nonsusceptible isolates respectively, compared to meropenem (100\% and $55.2 \%$ respectively) and imipenem $(100 \%$ and $58.6 \%$ respectively). Among P. aeruginosa isolates, doripenem had susceptibility rates of $84.2 \%$ and $26.7 \%$ against ceftazidime-susceptible and non-susceptible isolates respectively, compared to meropenem $(89.5 \%$ and $20 \%$ respectively) and imipenem ( $94.7 \%$ and $20 \%$ respectively). In Acinetobacter spp., doripenem had susceptibility rates of $84.2 \%$ and $26.7 \%$ against ceftazidime-susceptible and nonsusceptible isolates respectively, compared to meropenem (89.5\% and $20 \%$ respectively) and imipenem (94.7\% and $20 \%$ respectively).

\begin{tabular}{|c|c|c|c|}
\hline Antimicrobial Agent Tested & $\mathbf{\% ~ S}^{*}$ & $\mathbf{\% ~ I ~}^{\dagger}$ & $\mathbf{\% ~ R}^{\ddagger}$ \\
\hline Doripenem & 55.8 & 0.4 & 43.8 \\
\hline Imipenem & 63.7 & 3.2 & 33.1 \\
\hline Meropenem & 65.5 & 4.8 & 29.7 \\
\hline Piperacillin tazobactam & 48.7 & 3.3 & 48 \\
\hline Ceftazidime & 26.9 & 4.0 & 69.1 \\
\hline Ceftriaxone & 23.9 & 1.8 & 74.3 \\
\hline Amikacin & 53.4 & 1.8 & 44.8 \\
\hline Ciprofloxacin & 25.3 & 0.8 & 73.9 \\
\hline Trimethoprim-sulphamethoxazole & 38.3 & 0.4 & 61.3 \\
\hline Table 1: Antimicrobial Susceptibility \\
Patterns of Gram Negative Bacteria \\
\hline \multicolumn{4}{|l}{} \\
\hline
\end{tabular}

*S Susceptible, + I Intermediate, ₹ R Resistant 


\begin{tabular}{|c|c|c|c|c|c|c|c|}
\hline Antimicrobials & $\begin{array}{c}\text { E. coli } \\
\text { (n=157) } \\
(\%)\end{array}$ & $\begin{array}{c}\text { Klebsiella spp. } \\
(n=97) \\
(\%)\end{array}$ & $\begin{array}{c}\text { Enterobacter } \\
\text { spp. } \\
(n=41) \\
(\%)\end{array}$ & $\begin{array}{c}P . \\
\text { aeruginosa } \\
(n=83) \\
(\%)\end{array}$ & $\begin{array}{c}\text { Acinetobacter } \\
\text { spp. } \\
(n=100) \\
(\%)\end{array}$ & $\begin{array}{c}\text { Proteus spp. } \\
(n=15) \\
(\%)\end{array}$ & $\begin{array}{c}\text { Citrobacter } \\
\text { spp. } \\
(n=5) \\
(\%)\end{array}$ \\
\hline Doripenem & 83.4 & 40.2 & 75.6 & 53.0 & 14.1 & 86.7 & 80 \\
\hline Imipenem & 85.4 & 59.8 & 73.2 & 51.8 & 33,0 & 100 & 60 \\
\hline Meropenem & 89.2 & 64.9 & 68.3 & 50.6 & 31.0 & 93.3 & 100 \\
\hline Pip/taz* & 75.0 & 26.6 & 51.4 & 53.1 & 15.2 & 93.3 & 80 \\
\hline Ceftazidime & 31.8 & 16.5 & 29.3 & 45.8 & 05.0 & 66.7 & 60 \\
\hline Ceftriaxone & 29.3 & 14.4 & 22.0 & 39.8 & 04.0 & 66.7 & 60 \\
\hline Amikacin & 82.8 & 35.1 & 61.0 & 59.0 & 11.0 & 86.7 & 80 \\
\hline Ciprofloxacin & 21.7 & 23.7 & 22.0 & 45.8 & 04.0 & 80.0 & 40 \\
\hline Tmp-smx ${ }^{\dagger}$ & 33.8 & 93.8 & 24.4 & 10.8 & 06.0 & 20.0 & 20 \\
\hline
\end{tabular}

\begin{tabular}{|c|c|c|c|c|c|c|}
\hline & $\begin{array}{c}\text { Total } \\
(n=71)\end{array}$ & $\begin{array}{l}\text { E. coli } \\
(n=4) \\
(\%)\end{array}$ & $\begin{array}{c}\text { Klebsiella spp. } \\
(n=21) \\
(\%)\end{array}$ & $\begin{array}{c}\text { Enterobacter } \\
\text { spp. }(\mathrm{n}=7) \\
(\%)\end{array}$ & $\begin{array}{c}\text { Pseudomonas } \\
\text { spp. (n=9) } \\
(\%)\end{array}$ & $\begin{array}{c}\text { Acinetobacter } \\
\text { spp. }(n=30) \\
(\%)\end{array}$ \\
\hline Doripenem & 26.8 & 75 & 33.3 & 57.1 & 11.1 & 13.3 \\
\hline Imipenem & 39.4 & 75 & 57.1 & 57.1 & 22.2 & 23.3 \\
\hline Meropenem & 45.1 & 75 & 47.6 & 57.1 & 66.7 & 30 \\
\hline $\begin{array}{l}\text { Piperacillin } \\
\text { tazobactam }\end{array}$ & 18.8 & 100 & 15 & 42.9 & 33.3 & 6.7 \\
\hline Ceftazidime & 12.7 & 50 & 9.5 & 28.6 & 22.2 & 3.3 \\
\hline Ceftriaxone & 8.5 & 25 & 9.5 & 14.3 & 11.1 & 3.3 \\
\hline Amikacin & 22.5 & 75 & 23.8 & 14.3 & 55.6 & 3.3 \\
\hline $\begin{array}{c}\text { Trimethoprim- } \\
\text { sulphamethoxazole }\end{array}$ & 9.9 & 0 & 23.8 & 14.3 & 0 & 3.3 \\
\hline Ciprofloxacin & 7.1 & 25 & 10 & 14.3 & 11.1 & 0 \\
\hline
\end{tabular}

Figure: Antimicrobial activity of doripenem, imipenem and meropenem against Ceftazidime susceptible and nonsusceptible isolates
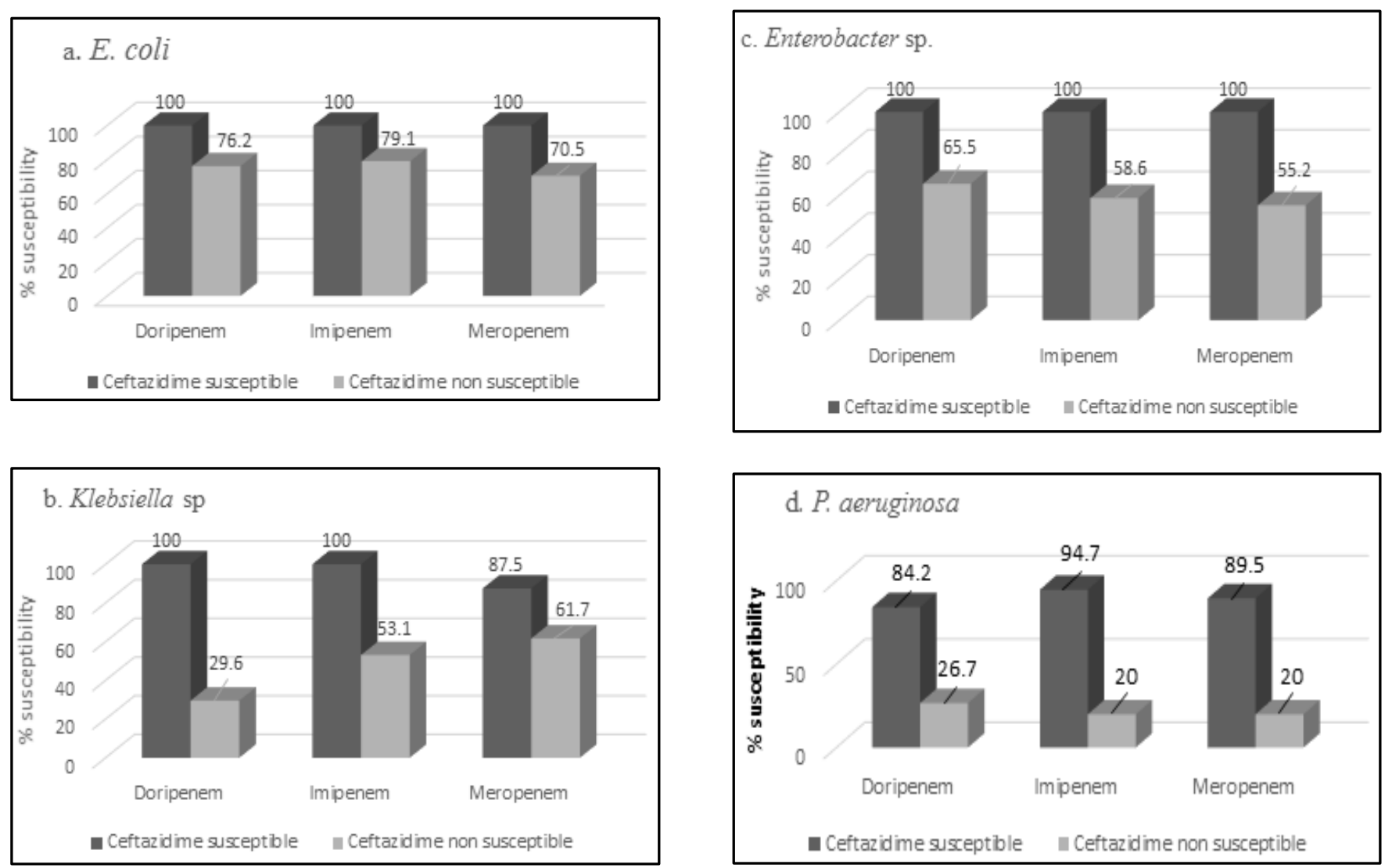


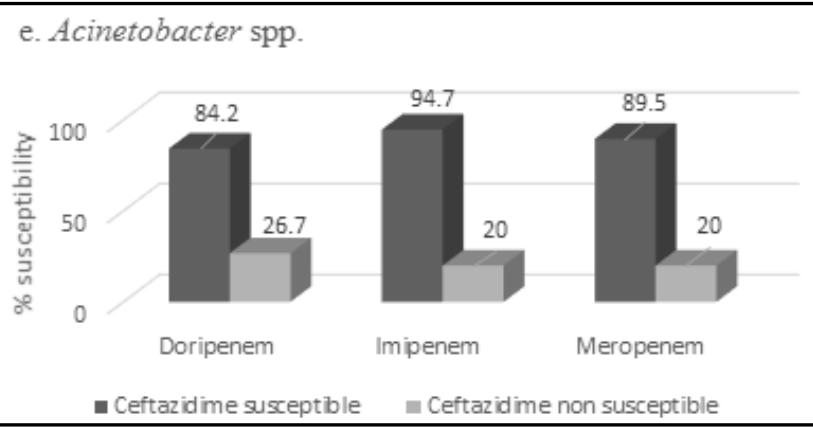

\section{DISCUSSION}

This retrospective study demonstrated that doripenem has similar or slightly lower activity than imipenem and meropenem against all the gram negative bacterial isolates tested except for Klebsiella pneumoniae and Acinetobacter spp. This pattern of activity for doripenem was similar to that observed in previous studies. (7-10) The rates of susceptibility to carbapenems seen in this study were low, which is comparable to other studies conducted in India. Wattal et al reported resistance rates of $13 \%, 51 \%, 59 \%$ and $80 \%$ among E. coli, Klebsiella spp., Pseudomonas spp. and Acinetobacter spp. respectively in Delhi.(11) Another study from a tertiary care hospital in North India reported resistance rates ranging from 17-22\% among Enterobacteriaceae, Pseudomonas spp. and Acinetobacter spp.(12)

Among the Enterobacteriaceae, majority of isolates of E. coli $(83.4 \%)$ and Enterobacter spp. (75.6\%) showed good susceptibility to doripenem, while lower rates of sensitivity were observed in Klebsiella spp. (40.2\%). According to CDC, the resistance rates in isolates belonging to Enterobacteriaceae to carbapenems increased from $0 \%$ in 2001 to $1 \cdot 4 \%$ in $2010 .(1,13)$ There is scarcity of studies reporting prevalence of carbapenem resistance among Enterobacteriaceae in India. In this study, 59.8\% and $64.9 \%$ of Klebsiella spp. showed susceptibility to imipenem and meropenem respectively. In a study conducted among 105 Enterobacteriaceae isolates from blood of septicaemic neonates, $74 \%$ of E. coli and $91 \%$ of Klebsiella pneumoniae were found to be susceptible to meropenem.(14) Shraddha et al reported resistance rates of $58.82 \%$ and $8.82 \%$ to meropenem and imipenem among E. coli and resistance rates of 53.84\% and $30.76 \%$ to meropenem and imipenem among Klebsiella pneumoniae respectively.(15) In a study from Korea, Choi et al reported low resistance rates among Enterobacteriaceae (E. coli - 0\%, Klebsiella spp. - 5.1\% and Enterobacter spp. - 0\%) to doripenem.(16)

The activity of all the three carbapenems against Pseudomonas spp. was comparable to each other. The nonsusceptible (Intermediate and resistant) rate of $48.2 \%$ for P. aeruginosa to imipenem observed in this study is higher than that observed in other studies.(17-19) There was a significant difference in activity of doripenem, imipenem and meropenem against ceftazidime susceptible and ceftazidime nonsusceptible isolates of Pseudomonas spp. This illustrates that some but not all of the mechanisms conferring resistance to cephalosporins in P. aeruginosa also confer resistance to carbapenems. In P. aeruginosa, resistance to doripenem can arise from a combination of loss of OprD protein and increased expression of pump efflux. None of the 3 carbapenems showed good activity against Acinetobacter spp.
In a study conducted in South India, high resistance rates of $100 \%$ and $89 \%$ to imipenem and meropenem in 55 isolates of A. baumannii were reported.(20) In North India, Sinha et al reported $14 \%$ meropenem resistance, Taneja et al reported $18.5 \%$ imipenem resistance and Mahajan et al reported $31.81 \%$ meropenem resistance in Acinetobacter isolates.(17,21,22) Castanheira et al reported higher susceptibility rates among 3,844 A. baumannii complex isolates to imipenem and meropenem in comparison with doripenem (Imipenem-69.4\%, meropenem-66.6\% and doripenem 49.9\%).(23) Production of metallo-beta-lactamase or OXA-type carbapenemases in Acinetobacter spp. may be responsible for reduced susceptibility to doripenem as well as other carbapenems in this study. $(23,24)$

Few publications have reported the comparative activity of doripenem in comparison with meropenem and imipenem against commonly isolated Gram negative bacilli from India. In this study, the activity of doripenem was lower than meropenem against all the isolates tested. Further detailed evaluation of doripenem is required with in-vitro MIC studies and their correlation with clinical outcomes.

\section{REFERENCES}

1. Jesse T Jacob, Eili Klein, Ramanan Laxminarayan, et al. Vital signs: carbapenem-resistant enterobacteriaceae. Morbidity and mortality weekly report 2013;62(9):16570.

2. Paterson DL, Depestel DD. Doripenem. Clinical infectious diseases 2009;49(2):291-8.

3. Jones RN, Huynh HK, Biedenbach DJ. Activities of doripenem (S-4661) against drug-resistant clinical pathogens. Antimicrobial agents and chemotherapy 2004;48(8):3136-40.

4. Jones RN, Huynh HK, Biedenbach DJ, et al. Doripenem (S4661), a novel carbapenem: comparative activity against contemporary pathogens including bactericidal action and preliminary in vitro methods evaluations. The Journal of antimicrobial chemotherapy 2004;54(1):14454.

5. Murray PR, Baron, EJ, Jorgensen JH, et al. Manual of clinical microbiology. Washington DC: ASM Press 2003; $8^{\text {th }}$ ed.

6. Performance standards for antimicrobial susceptibility testing: 22th informational supplement. Wayne, PA: Clinical and laboratory standards institute, 2012.

7. Fritsche TR, Stilwell MG, Jones RN. Antimicrobial activity of doripenem (S-4661): a global surveillance report (2003). Clinical microbiology infect 2005;11(12):97484.

8. Jones RN, Sader HS, Fritsche TR. Comparative activity of doripenem and three other carbapenems tested against Gram-negative bacilli with various beta-lactamase resistance mechanisms. Diagnostic microbiology and infectious disease 2005;52(1):71-4.

9. Ge Y, Wikler MA, Sahm DF, et al. In vitro antimicrobial activity of doripenem, a new carbapenem. Antimicrobial agents and chemotherapy 2004;48(4):1384-96.

10. Mushtaq S, Ge Y, Livermore DM. Comparative activities of doripenem versus isolates, mutants, and transconjugants of enterobacteriaceae and acinetobacter spp. with characterized beta-lactamases. Antimicrobial agents and chemotherapy 2004;48(4): 1313-9. 
11. Wattal C, Goel N, Oberoi JK, et al. Surveillance of multidrug resistant organisms in tertiary care hospital in Delhi, India. The Journal of the Association of Physicians of India 2010;58:32-6.

12. Gupta E, Mohanty S, Sood S, et al. Emerging resistance to carbapenems in a tertiary care hospital in north India. The Indian journal of medical research 2006;124(1): 95-8.

13. Laxminarayan R, Duse A, Wattal C, et al. Antibiotic resistance-the need for global solutions. Lancet Infectious diseases 2013;13(12):1057-98.

14. Datta S, Roy S, Chatterjee S, et al. A five-year experience of carbapenem resistance in Enterobacteriaceae causing neonatal septicaemia: predominance of NDM-1. PloS one 2014;9(11):e112101.

15. Shraddha Prasad Gunjal, Nagaraju Vanaparthi, Kher Sudhee. Carbapenem resistance profile amongst escherichia coli and klebsiella pneumoniae in a tertiary care hospital in ahmednagar, Maharashtra. International Journal of Medical Research \& Health Sciences 2014;3(2):424-27.

16. Choi SH, Ahn MY, Chung JW, et al. In vitro antibacterial activity of doripenem against gram-negative blood isolates in a Korean tertiary care center. Infection chemotherapy 2015;47(3):175-80.

17. Taneja N, Maharwal S, Sharma M. Imipenem resistance in nonfermenters causing nosocomial urinary tract infections. Indian journal of medical sciences 2003;57(7):294-9.

18. Gladstone P, Rajendran P, Brahmadathan KN. Incidence of carbapenem resistant nonfermenting gram negative bacilli from patients with respiratory infections in the intensive care units. Indian journal of medical microbiology 2005;23(3):189-91.
19. Sharma M, Yadav S, Chaudhary U. Metallo-betalactamase producing pseudomonas aeruginosa in neonatal septicemia. Journal of laboratory physicians 2010;2(1):14-6.

20. Uma Karthika R, Srinivasa Rao R, Sahoo S, et al. Phenotypic and genotypic assays for detecting the prevalence of metallo-beta-lactamases in clinical isolates of acinetobacter baumannii from a south Indian tertiary care hospital. Journal of medical microbiology 2009;58(Pt 4):430-5.

21. Mahajan G, Sheemar S, Chopra S, et al. Carbapenem resistance and phenotypic detection of carbapenemases in clinical isolates of acinetobacter baumannii. Indian journal of medical sciences 2011;65(1):18-25.

22. Sinha N, Agarwal J, Srivastava S, et al. Analysis of carbapenem-resistant acinetobacter from a tertiary care setting in North India. Indian journal of medical microbiology 2013;31(1):60-3.

23. Castanheira M, Jones RN, Livermore DM. Antimicrobial activities of doripenem and other carbapenems against pseudomonas aeruginosa, other nonfermentative bacilli, and aeromonas spp. Diagnostic microbiology and infectious disease 2009;63(4):426-33.

24. Terzi HA, Atasoy AR, Aykan SB, et al. Association of doripenem resistance with OXA-type carbapenemases in Acinetobacter baumannii isolates. Saudi medical journal 2016;37(1):43-7. 